\title{
THEORETICAL ANALYSIS OF FILM UNIFORMITY IN SPINNING PROCESSES
}

\author{
CHIH-TA WANG and SHI-CHERN YEN ${ }^{\dagger}$ \\ Department of Chemical Engineering, National Taiwan University, Taipei, Taiwan 106
}

(First received 27 May 1994; accepted in revised form 17 October 1994)

\begin{abstract}
The coating film uniformity of a Newtonian fluid on a spinning disc is analyzed theoretically. The effect of evaporation during the spin coating is also investigated. In this study the perturbation technique has been applied to solve the nonlinear governing equation with or without evaporation, and it can be applied for any initial film thickness contour. The perturbation solution is explicit in film thickness. A dynamic uniformity factor is defined to describe the degree of uniformity with time evolution. In this study a criterion for the effect of evaporation on achieving film uniformity is proposed. It is shown theoretically for the case of small evaporation that the coating film thickness is getting more uniform across the whole disc with increasing spinning time.
\end{abstract}

\section{INTRODUCTION}

For the thin film formation on a spinning disc, two methods, depending on the different ways of transporting fluid onto the disc, could be employed. One way of forming a thin solidified film, as seen in Fig. 1, is that a fixed volume of process fluid is first placed on a stationary disc, forming a puddle on it because of surface tension, and then the disc is spun to a low rotating speed for several seconds and a high rotating speed for tens of seconds sequentially. The other way, called dynamic dispense, is to spread the coating fluid onto a disc spinning at a low speed, forming a liquid film to wet the whole disc, and then rapidly accelerate the disc to a preset speed. In each case the volume of fluid on the spinning disc decreases with increasing spinning time because of radial flows by centrifugal forces, and finally a thin film forms on the surface. This method is employed in microelectronic fabrications and other industries involving coating a thin film on a substrate, and is generally named spin coating. There are many ways to design delivery system to make various initial film profile in spin coating, and the aim of each method is to produce an uniform thin film on substrates. In lithography, a thin film called photoresist must be first applied on a wafer by spincoating technology. Exposure to appropriate radiation for a positive photoresist will result in dissociation of the bonding in polymers. Following the exposure, the wafer is washed with a solvent that preferentially removes the region of higher solubility and then is spun to dry. Finally etching the portions unprotected by the resist leaves a duplicate pattern of photomask on the wafer (Thompson and Bowden, 1983). In a multilevel metallization process, a polyimide solution or any other organic or inorganic viscous solution is spread by spinning on the underlying topo-

${ }^{\dagger}$ Author to whom correspondence should be addressed. graphy formed from the last processing step, and then is dried as an interlevel dielectric to generate more planar surface so that the sequential thin metal layer will not crack to disconnect the devices (Sze, 1988). In the method of spin-on doping, such as for arsenic or antimony, the doped oxide dissolved in some organic solvent is distributed uniformly on a wafer by a spinning process. In the manufacture of magnetic hard disk devices for data storage, lubricants must be spun on hard disks to avoid damage.

The thinning of liquid films results from two mechanisms. One is the radial convective flow outward due to centrifugal force, the other is evaporation of the solvent. Emslie et al. (1958) first analyzed the film forming of a nonevaporating Newtonian fluid on a flat spinning disc. They tested three profiles numerically and found that the film can be finally smoothed out after a sufficient time. Acrivos et al. (1960) considered the same problem, but introduced a powerlaw fluid and found a power-law fluid may never reach a layer of uniform thickness. Jenekhe and Schuldt $(1984,1985)$ made an extensive investigation on the film forming of several kinds of nonevaporating fluids, such as a power-law fluid, a Carreau fluid and a Bingham fluid, on a spinning disc. Several authors investigated the effect of inertia on nonevaporating film flow on a rotating disc by expanding the dynamic film in square of Reynolds number (Higgins, 1986) or by computing numerically by employing finite difference method (Rehg and Higgins, 1988). They assumed uniform film thinning, so radial dependence on film thickness is factored out. For film flow of small Reynolds number, the assumptions used by Emslie et al. (1958) are proved to be valid. But for a thick film flow, the effect of inertia becomes important. For a more accurate description of spin-coating process, the evaporation of solution must be taken into account. Lawrence $(1988,1989)$ investigated the uniform thinning of evaporating 


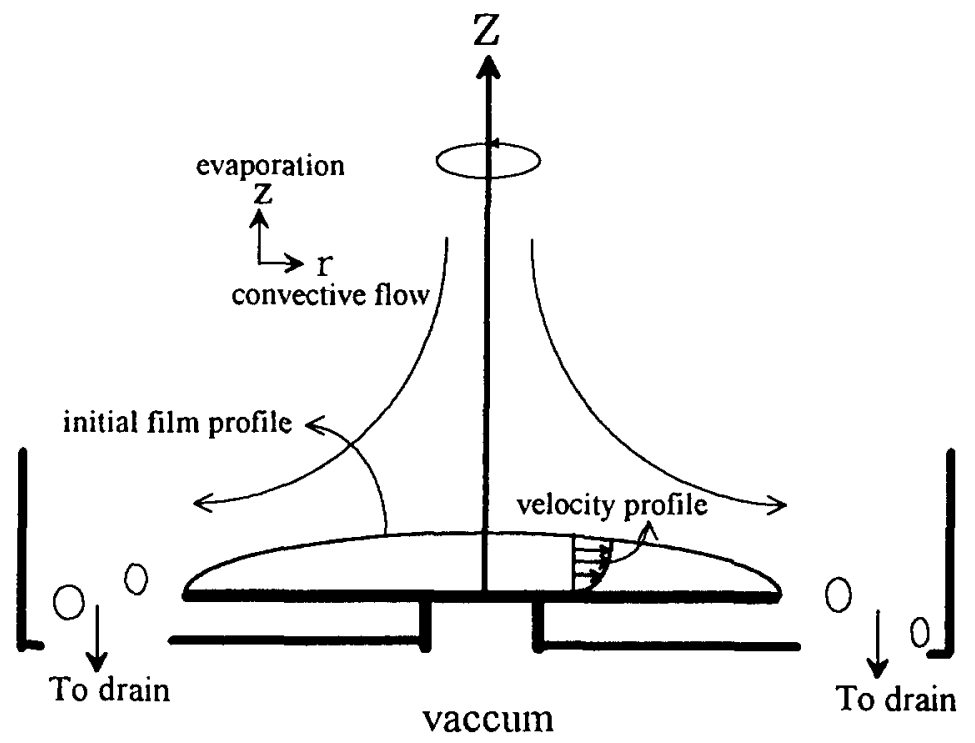

Fig. 1. A schematic diagram for film formation on a spinning disc.

liquid film based on the assumption of a rapid evaporation rate and thus a constant concentration at film surface, or a constant small evaporation rate determined by the mass transfer in the adjacent atmosphere and obtained a self-similar solution in each case. In practice evaporation proceeds during the film-forming process, and it makes the mathematical modeling more complicated, because the fluid properties, such as the viscosity and solvent fraction of the fluid, may change drastically with time. For the case of time-varying physical properties in spin coating, several researchers have studied numerically by focusing on the case of uniform initial film thickness across the flat spinning disc as well as uniform mass transfer rate toward gas phase, and thus final film thickness is not dependent on radial position (Washo, 1977; Meyerhofer, 1978; Flack et al., 1984; Sukanek, 1984; Bornside et al., 1989). When the initial film varies with radial position, or the evaporation rate toward gas phase is not uniform, the film thickness is a function of radial location, and striation (Elliott and Hockey, 1979) on the substrate can be formed.

In this study, we have developed an analytical approximate formulation by perturbation technique for film uniformity, with or without evaporation. The perturbation solution is explicit in film thickness, so it can be used more conveniently to compute the film thickness. The perturbation solutions are proved to be accurate enough in comparison with the exact numerical solutions obtained by the finite difference method by testing two nonuniform initial film profiles. A criterion for the effect of solvent evaporation on achieving the film uniformity across the disc is also proposed in this study.

\section{THEORETICAL ANALYSIS}

We consider an isothermal (if evaporation occurs, we assume that the temperature variation is not large enough to affect the rheological properties and velocity profile), incompressible and homogeneous Newtonian fluid that wets completely the whole spinning disc. The system is assumed to have cylindrical symmetry. The governing equation can be derived by employing the assumption of low Reynolds number resulting from thin film thickness and simplifying the complete equation of motion in cylindrical coordinates, thus giving

$$
\begin{gathered}
\mu \frac{\partial^{2} v_{r}}{\partial z^{2}}-\frac{\partial p}{\partial r}=-\rho \frac{v_{\theta}^{2}}{r} \\
\frac{\partial^{2} v_{\theta}}{\partial z^{2}}=0
\end{gathered}
$$

where $\rho$ is the density of this process fluid, $v_{\theta}$ is the azimuthal velocity, $\omega$ is the angular velocity of the spinning disc, $z$ is the axial distance from the surface of the spinning disc, $r$ is the radial distance from the center of the spinning disc, $\mu$ is the viscosity of the fluid and $v_{r}$ is the radial velocity. The boundary conditions for this problem are

$$
\begin{gathered}
z=0, \quad v_{\mathrm{r}}=0, \quad v_{\theta}=r \omega \\
z=h, \quad-\mu \frac{\mathrm{d} v_{r}}{\mathrm{~d} z}=\frac{\mathrm{d} \sigma}{\mathrm{d} r}, \quad \frac{\mathrm{d} v_{\theta}}{\mathrm{d} z}=0
\end{gathered}
$$

where $\sigma$ is the surface tension. The first boundary condition for film flow is the no-slip condition at the disc-film surface, and the second describes the tangential shear stress must be equal to the gradient of surface tension at the topographic film-air interface. Solving the governing equation, eq. (1), with the boundary condition, eq. (2), the radial velocity distribution can be obtained. Then the volumetric flow rate $q$ per circumference can be shown to be

$$
q=\int_{0}^{h} v_{r} \mathrm{~d} z=\left(\rho \omega^{2} r-\frac{\partial p}{\partial r}\right) \frac{h^{3}}{3 \mu}+\frac{h^{2}}{2 \mu} \frac{\mathrm{d} \sigma}{\mathrm{d} r} .
$$


In addition we need the equation of Young and Laplace to describe the relationship between pressure and surface tension (Adamson, 1982). The kinematic boundary condition at free surface can be obtained from making mass balance at $z=h$, and is given by (Bornside et al., 1987)

$$
\frac{\partial h}{\partial t}=v_{z}-v_{r} \frac{\partial h}{\partial r}-\frac{m}{\rho} \sqrt{1+\left(\frac{\partial h}{\partial r}\right)^{2}}
$$

where $m$ is the mass flux from process liquid to gaseous phase due to the evaporation of liquid. Because the disc is nonporous, and no liquid can penetrate, the axial velocity is zero at the disc surface. The local continuity equation can be averaged by integrating over the film thickness $[0, h]$. Combined with the kinematic boundary condition at free surface and the boundary condition at disc-solution surface, the axial average film thickness is given by (Bornside, et al., 1987)

$$
\frac{\partial h}{\partial t}+\frac{1}{r} \frac{\partial(r q)}{\partial r}+\frac{m}{\rho}=0
$$

where $h=h(r, t)$ represents film thickness which is a function of position and time. In a photoresist solution, nonvolatile polymers are dissolved in volatile solvents. So $m$ is only attributed to solvent evaporation and depends on the fraction of solvent in the film. It can be approximated by a pure rotating disc behaviour, which is proportional to the square root of spinning speed in the laminar flow region (Levich, 1962). For a low Schmidt number gas flow on a rotating disc, a correction factor is obtained (Yen and Wang, 1992). In practical situations $m$ decreases with spinning time, because the fraction of solvent decreases with time and finally vanishes, but here $m$ is assumed to be a constant. The gradients of the surface tension and pressure in radial direction due to small radial variation in film thickness are assumed to be negligible. The following dimensionless variables are introduced to make eq. (5) dimensionless:

$$
H=\frac{h}{h_{0}} ; \quad R=\frac{r}{r_{0}} ; \quad T=\frac{t}{t_{0}} ; \quad t_{0}=\frac{\mu}{h_{0}^{2} \rho \omega^{2}}
$$

where $h_{0}, r_{0}$, and $t_{0}$ are all characteristic scaling factors. $r_{0}$ is the radius of the disc, and $h_{0}$ the reference initial film thickness on the disc. Here $t_{0}$ is a time parameter. With the substitution of eq. (6), eq. (5) can be made dimensionless to become

$$
\frac{\partial H}{\partial T}+R H^{2} \frac{\partial H}{\partial R}+\frac{2}{3} H^{3}+M=0
$$

where $M$ represents physically the ratio of mass flux resulting from evaporation to the strength of radial convective outflow, and is expressed as

$$
M=\frac{m t_{0}}{\rho h_{0}}=\frac{m \mu}{\rho^{2} \omega^{2} h_{0}^{3}} .
$$

If a high volatile solvent is employed $M$ will be relatively large. For a laminar flow the mass transfer rate $m$ is proportional to the square root of the spinning speed $\omega$, so $M$ would be inversely proportional to the one and a half power of the spinning speed. Hence $M$ decreases as the spinning speed increases.

For the case of film formation without evaporation, $M$ is zero, and thus eq. (7) is reduced to be a homogeneous nonlinear differential equation which can be solved analytically by the Lagrange method (Aris and Amundson, 1973). Its general solution is expressed as

$$
\frac{1}{2 H^{2}}-\frac{2}{3} T=f^{*}\left(H R^{2 / 3}\right)
$$

where $f^{*}$ is an arbitrary function that is exactly determined by the initial condition. In combination with the initial condition of uniform film thickness on the disc, one obtains

$$
H=\left(\frac{1}{1+\frac{4}{3} T}\right)^{1 / 2}
$$

If the initial film thickness varies with radial position, implicit exact analytical solution can be derived by expanding the initial profile in a power series. But for any arbitrary radially distributed function, the exact solution becomes very complicated and thus tedious in application. Here we employ the perturbation method to develop the general approximate solution for the case of initial nonuniform film. For small radial variations in the initial film profile, the initial condition can be expressed as

$$
H(R, 0)=H^{*}(R)=1+\xi g(R)
$$

where $\xi$ is a small parameter, which can be defined by the initial degree of radial deviation of the liquid film. $g(R)$ is an arbitrary function that can describe the initial film profile. So the general solution can be expressed by using $\xi$ as a perturbation quantity in series expansion, and is given as

$$
H=H_{0}+\xi H_{1}+\xi^{2} H_{2}+\cdots
$$

Substitute eq. (12) into the governing equation to generate the differential equations for $H_{0}, H_{1}$ and $H_{2}$ by taking the coefficient of $\xi^{i}$ to be zero. The zeroorder solution, $H_{0}$, is unchanged and is given by eq. (10). The partial differential equation for the firstorder and the second-order terms, $H_{1}$ and $H_{2}$ are, respectively, taken to be

$$
\begin{aligned}
\frac{\partial H_{1}}{\partial T} & +R H_{0}^{2} \frac{\partial H_{1}}{\partial R}+2 R H_{0} H_{1} \frac{\partial H_{0}}{\partial R}+2 H_{0}^{2} H_{1}=0 \\
\frac{\partial H_{2}}{\partial T}+ & R H_{0}^{2} \frac{\partial H_{2}}{\partial R}+R\left(2 H_{0} H_{2}+H_{1}^{2}\right) \frac{\partial H_{0}}{\partial R} \\
& +2 R H_{0} H_{1}^{2} \frac{\partial H_{1}}{\partial R}+2 H_{0}^{2} H_{2}+2 H_{1}^{2} H_{0}=0 .
\end{aligned}
$$

The approximate solution by employing the perturbation technique in two-term expansion is thus obtained to be

$$
\begin{aligned}
H= & \left(\frac{3}{3+4 T}\right)^{1 / 2}+\xi \times\left(\frac{3}{3+4 T}\right)^{3 / 2} \\
& \times g\left[\left(\frac{3}{3+4 T}\right)^{3 / 4} R\right]+\mathrm{O}\left(\xi^{2}\right) .
\end{aligned}
$$


So when the arbitrary function $g(R)$ is known, the approximate perturbation solutions in two-term expansion can be easily obtained from the above equation, eq. (14), for any profile. The perturbation solutions of second and higher powers of $\xi$ can also be obtained if the characteristic equation of each corresponding partial differential equation is intergrable analytically. For example, the complete solution in three-term expansion with this linear initial condition, $g(R)=-R$, is solved to be

$$
\begin{aligned}
H= & \left(\frac{3}{3+4 T}\right)^{1 / 2}-\xi\left(\frac{3}{3+4 T}\right)^{9 / 4} R \\
& +3 \xi^{2} R^{2}\left(\frac{3}{3+4 T}\right)^{3}\left[\left(\frac{3}{3+4 T}\right)-1\right]+\cdots
\end{aligned}
$$

If the initial surface contour varies periodically with radial position, as described by a sinusoidal function $g(R)=-\sin (2 \pi k R)$ the complete solution in threeterm expansion is

$$
H=H_{0}+\xi H_{1}+\xi^{2} H_{2}+\cdots
$$

where

$$
\begin{aligned}
H_{0}= & \left(\frac{3}{3+4 T}\right)^{1 / 2} \\
H_{1}= & -\left(\frac{3}{3+4 T}\right)^{3 / 2} \sin \left[2 \pi k\left(\frac{3}{3+4 T}\right)^{3 / 4} R\right] \\
H_{2}= & \frac{12}{7} \pi k \sin \left[2 \pi k\left(\frac{3}{3+4 T}\right)^{3 / 4} R\right] \\
& \times \cos \left[2 \pi k\left(\frac{3}{3+4 T}\right)^{3 / 4} R\right] \\
& \times\left[\left(\frac{3}{3+4 T}\right)^{13 / 4}-\left(\frac{3}{3+4 T}\right)^{3 / 2}\right] \\
& +\frac{3}{4} \sin ^{2}\left[2 \pi k\left(\frac{3}{3+4 T}\right)^{3 / 4} R\right]\left[\left(\frac{3}{3+4 T}\right)^{5 / 2}\right. \\
& \left.-\left(\frac{3}{3+4 T}\right)^{3 / 2}\right] .
\end{aligned}
$$

When spin-coating technology is applied in microlithography, the process liquid is composed of nonvolatile polymers dissolved in volatile organic solvents. During the process of spin coating the viscosity of the polymer solution increases drastically due to the evaporation of solvents, which complicates the complete mathematical modeling on spin coating. In addition to the overall material balance, the mass balance for each constituent in the solution, the overall energy balance in the liquid film, and the temperature- and concentration-dependent viscosity must be incorporated into the mathematical model. But in this theoretical analysis we investigate the case that the system parameter, such as the viscosity, is supposed to be constant. In other words the corresponding increasing viscosity of the coating film is neglected. This assumption is only suitable for the case of small Biot number, i.e. small ratio of internal resistance to the external resistance. In this case, for example the early stage in coating the diluted low vapor pressure solution, the evaporation rate is sufficiently small and thus the viscosity is assumed uniform within the film. More accurate models with uniform film on the rotating disk that include the variation of viscosity can be obtained (Sukanek, 1985, Bornside et al., 1989). The characteristic equation of the first-order nonlinear partial differential equation, eq. (7), is

$$
\frac{\mathrm{d} T}{1}=\frac{\mathrm{d} R}{R H^{2}}=\frac{\mathrm{d} H}{\frac{2}{3} H^{3}+\varepsilon^{3}}
$$

where $\varepsilon=M^{1 / 3}$. Following the same procedure as taken in the last case, the form of general solution to eq. (7) for film formation with evaporation can be expressed as

$$
\begin{array}{r}
a T+\frac{1}{6 \varepsilon^{2}} \ln \left[\frac{(a H+\varepsilon)^{2}}{a^{2} H^{2}-a \varepsilon H+\varepsilon^{2}}\right]+\frac{1}{\sqrt{3} \varepsilon^{2}} \tan ^{-1} \frac{a \sqrt{3}}{2 \varepsilon-a H} \\
=f^{*}\left[R^{2 / 3}\left(H^{3}+\frac{3}{2} \varepsilon^{3}\right)^{1 / 3}\right]
\end{array}
$$

where $a=(2 / 3)^{1 / 3}$. The second and third terms on the left-hand side of the above equation are the indefinite integral of $\left(\frac{2}{3} H^{3}+\varepsilon^{2}\right)^{-1}$. The function $f^{*}$ depends on the initial conditions. When the initial film thickness distributes uniformly, eq. (18) would be reduced to

$$
\begin{aligned}
a T= & \frac{1}{6 \varepsilon^{2}} \ln \left[\frac{(a+\varepsilon)^{2}}{a^{2}-a \varepsilon+\varepsilon^{2}}\right]+\frac{1}{\sqrt{3} \varepsilon^{2}} \tan ^{-1} \frac{a \sqrt{3}}{2 \varepsilon-a} \\
& -\frac{1}{6 \varepsilon^{2}} \ln \left[\frac{(a H+\varepsilon)^{2}}{a^{2} H^{2}-a \varepsilon H+\varepsilon^{2}}\right] \\
& -\frac{1}{\sqrt{3} \varepsilon^{2}} \tan ^{-1} \frac{a \sqrt{3}}{2 \varepsilon-a H} .
\end{aligned}
$$

This solution form can be easily reduced to eq. (10), the solution without evaporation, when $\varepsilon$ approaches zero. There are two limiting cases for the effect of evaporation:

(i) If the evaporation parameter $\varepsilon$ is small (i.e. $\left.3 \varepsilon^{3} / 2 H^{3}<1\right)$, the term $\left(\frac{2}{3} H^{3}+\varepsilon^{3}\right)^{-1}$ in eq. (17) can be expressed in power series, and then integrated to obtain the approximate solution subject to nonuniform initial film thickness

$$
\begin{aligned}
\frac{1}{2 H^{2}}\left[1-\frac{3}{5} \frac{\varepsilon^{3}}{H^{3}}+\frac{9}{16}\right. & \left.\frac{\varepsilon^{6}}{H^{6}}-\cdots\right]-\frac{2}{3} T \\
& =f^{*}\left[R^{2 / 3}\left(H^{3}+\frac{3}{2} \varepsilon^{3}\right)^{1 / 3}\right]
\end{aligned}
$$

which can be reduced to eq. (10) for $\varepsilon=0$.

(ii) If the evaporation parameter $\varepsilon$ is large (i.e. $\left(2 H^{3} / 3 \varepsilon^{3}<1\right)$, then by using the same procedures, the approximate solution is

$$
\begin{aligned}
\varepsilon^{-3} H\left[1-\frac{1}{6} \frac{H^{4}}{\varepsilon^{4}}+\right. & \left.\frac{4}{63} \frac{H^{7}}{\varepsilon^{7}}-\cdots\right]+T \\
& =f^{*}\left[R^{2 / 3}\left(H^{3}+\frac{3}{2} \varepsilon^{3}\right)^{1 / 3}\right] .
\end{aligned}
$$


It is found from the above formula that the film uniformity cannot be improved with spinning time.

From these two cases, a criterion for achieving the film uniformity can be proposed as

$$
M=\varepsilon^{3}<\frac{2}{3} H_{\min }^{3} \text {, initial }
$$

where $H_{\text {min, initial }}$ is the minimum dimensionless thickness at initial time. If the evaporation parameter $M\left(=\varepsilon^{3}\right)$ is less than $\frac{2}{3} H_{\min , \text { initial }}^{3}$, the film uniformity will be improved.

For the effect of small evaporation the perturbation solution to eq. (7) can be expressed in power series of the evaporation parameter $\varepsilon^{3}$.

$$
H=H_{0}+\varepsilon^{3} H_{1}+\varepsilon^{6} H_{2}+\cdots
$$

The partial differential equation in this case is shown to be as follows for the zero-order, first-order and second-order solution, respectively:

$$
\begin{aligned}
& \frac{\partial H_{0}}{\partial T}+R H_{0}^{2} \frac{\partial H_{0}}{\partial R}+\frac{2}{3} H_{0}^{3}=0 \\
& \frac{\partial H_{1}}{\partial T}+R H_{0}^{2} \frac{\partial H_{1}}{\partial R}+2 R H_{1} H_{0} \frac{\partial H_{0}}{\partial R}+2 H_{0}^{2} H_{1}+1=0 \\
& \frac{\partial H_{2}}{\partial T}+R\left[\left(H_{1}^{2}+2 H_{0} H_{2}\right) \frac{\partial H_{0}}{\partial R}+2 H_{0} H_{1} \frac{\partial H_{1}}{\partial R}\right. \\
& \left.\quad+H_{0}^{2} \frac{\partial H_{2}}{\partial R}\right]+2\left(H_{0}^{2} H_{2}+H_{1}^{2} H_{0}\right)=0
\end{aligned}
$$

For the case of a uniform initial film the approximate solution in three-term expansion on spin coating with evaporation is

$$
\begin{aligned}
H= & \left(\frac{3}{3+4 T}\right)^{1 / 2}+\varepsilon^{3} \frac{1}{10}(3+4 T)\left[\left(\frac{3}{3+4 T}\right)^{5 / 2}-1\right] \\
& +\varepsilon^{6}(3+4 T)^{-3 / 2}\left\{\frac{\sqrt{3}}{200}\left(\frac{1}{3+4 T}-\frac{1}{3}\right)\right. \\
& +\frac{9}{50}\left[(3+4 T)^{3 / 2}-3^{3 / 2}\right] \\
& \left.+\frac{\sqrt{3}}{800}\left[3^{4}-(3+4 T)^{4}\right]\right\}+\cdots
\end{aligned}
$$

When the initial film thickness has a small radial variation, the initial thickness can also be described by eq. (11). In this case, $\xi$ is introduced to be the second perturbation quantity, and therefore $H_{0}$ and $H_{1}$ is assumed, respectively, to have expansion in the form

$$
\begin{aligned}
& H_{0}=H_{00}+\xi H_{01}+\cdots \\
& H_{1}=H_{10}+\xi H_{11}+\cdots
\end{aligned}
$$

Substituting eq. (26) into eq. (24) and equating the coefficient of each power of $\xi$ to be zero, we can obtain the partial differential equation of $H_{00}, H_{01}, H_{02}, H_{10}$, $H_{11}$ and $H_{20}$, respectively. In combination with the initial condition, the complete solution to the linearly distributed function of initial profile, $g(R)=-R$, is thus obtained

$$
\begin{aligned}
& H= H_{00}+\xi H_{01}+\xi^{2} H_{02}+\cdots+\varepsilon^{3}\left(H_{10}\right. \\
&\left.+\xi H_{11}+\cdots\right)+\varepsilon^{6}\left(H_{20}+\cdots\right)+\cdots \\
& H_{00}=\left(\frac{3}{3+4 T}\right)^{1 / 2} \\
& H_{01}=\left(\frac{3}{3+4 T}\right)^{9 / 4} R \\
& H_{02}= 3 R^{2}\left(\frac{3}{3+4 T}\right)^{3}\left[\left(\frac{3}{3+4 T}\right)-1\right] \\
& H_{10}= \frac{1}{10}(3+4 T)\left[\left(\frac{3}{3+4 T}\right)^{5 / 2}-1\right] \\
& H_{11}= \frac{9}{4} R\left(\frac{3}{3+4 T}\right)^{9 / 4}-\frac{27}{20} R\left(\frac{3}{3+4 T}\right)^{13 / 4} \\
&-\frac{9}{10} R\left(\frac{3}{3+4 T}\right)^{3 / 4} \\
& H_{20}=(3+4 T)^{-3 / 2}\left\{\frac{\sqrt{3}}{200}\left(\frac{1}{3+4 T}-\frac{1}{3}\right)\right. \\
&+\frac{9}{50}\left[(3+4 T)^{3 / 2}-3^{3 / 2}\right] \\
&\left.-\frac{\sqrt{3}}{800}\left[(3+4 T)^{4}-3^{4}\right]\right\} \\
&
\end{aligned}
$$

As the initial function is $g(R)=-\sin (2 \pi k R)$, the solution subject to this initial condition could be solved by the same procedure as that in the case dependent on radial position:

$$
\begin{aligned}
H= & H_{00}+\xi H_{01}+\xi^{2} H_{02}+\cdots+\varepsilon^{3}\left(H_{10}\right. \\
& \left.+\xi H_{11}+\cdots\right)+\varepsilon^{6}\left(H_{20}+\cdots\right)+\cdots \\
H_{00}= & \left(\frac{3}{3+4 T}\right)^{1 / 2} \\
H_{01}= & -\left(\frac{3}{3+4 T}\right)^{3 / 2} \sin \left[2 \pi k\left(\frac{3}{3+4 T}\right)^{3 / 4} R\right] \\
H_{02}= & \frac{12}{7} \pi k \sin \left[2 \pi k\left(\frac{3}{3+4 T}\right)^{3 / 4} R\right] \\
& \times \cos \left[2 \pi k\left(\frac{3}{3+4 T}\right)^{3 / 4} R\right] \\
& \times\left[\left(\frac{3}{3+4 T}\right)^{13 / 4}-\left(\frac{3}{3+4 T}\right)^{3 / 2}\right] \\
& +\frac{3}{4} \sin ^{2}\left[2 \pi k\left(\frac{3}{3+4 T}\right)^{3 / 4} R\right] \\
& \times\left[\left(\frac{3}{3+4 T}\right)^{5 / 2}-\left(\frac{3}{3+4 T}\right)^{3 / 2}\right] \\
H_{10}= & \frac{1}{10}(3+4 T)\left[\left(\frac{3}{3+4 T}\right)^{5 / 2}-1\right]
\end{aligned}
$$




$$
\begin{aligned}
H_{11}= & \pi k R \cos \left[2 \pi k\left(\frac{3}{3+4 T}\right)^{3 / 4} R\right] \\
& \times\left[-\frac{1}{30}\left(\frac{3}{3+4 T}\right)^{21 / 4}+\frac{1}{5}\left(\frac{3}{3+4 T}\right)^{1 / 4}\right. \\
& \left.+\frac{1}{6}\left(\frac{3}{3+4 T}\right)^{9 / 4}\right]+\sin \left[2 \pi k\left(\frac{3}{3+4 T}\right)^{3 / 4} R\right] \\
& \times\left[-\frac{1}{30}\left(\frac{3}{3+4 T}\right)^{9 / 2}+\frac{1}{5}\left(\frac{3}{3+4 T}\right)^{2}\right. \\
& \left.+\frac{1}{6}\left(\frac{3}{3+4 T}\right)^{3 / 2}\right] \\
H_{20}= & (3+4 T)^{-3 / 2}\left\{\frac{\sqrt{3}}{200}\left(\frac{1}{3+4 T}-\frac{1}{3}\right)+\frac{9}{50}\right. \\
& \times\left[(3+4 T)^{3 / 2}-3^{3 / 2}\right]-\frac{\sqrt{3}}{800} \\
& \left.\times\left[(3+4 T)^{4}-3^{4}\right]\right\}
\end{aligned}
$$

\section{RESULTS AND DISCUSSION}

As in the case for film formation of nonevaporating coating fluid on a spinning disc the general solution is represented by eq. (9), which indicates that the dynamic thickness distribution depends on the initial film contour. Therefore as long as the initial radial distribution is known, the dynamic film thickness can be found as a function of the radial position. The nonuniformity of the film thickness on a substrate is not allowed in microlithography because it will affect the success of sequential processing steps. For uniform initial film contour, the dynamic film thickness, as expressed in eq. (10), is obtained to be always uniform at any time. This is the best condition of employing the spin-coating technology in microlithography. By making eq. (10) to be dimensional, the dynamic film thickness is given by

$$
h=\sqrt{\frac{h_{0}^{2}}{\frac{4}{3} \rho \omega^{2} \mu h_{0}^{2} t+1}} .
$$

This solution is the same as that derived by Emslie $e t$ al. (1958), who considered conceptually that the film thickness was not a function of radial position, and thus an ordinary differential equation should be solved. If the disc spins very fast, eq. (29) can be reduced to

$$
h=\frac{\sqrt{3}}{2 \omega \sqrt{\rho \mu t}} .
$$

It is indicated from eq. (30) that the film thickness decreases with square root of time and is inversely proportional to the spinning speed of the disc, and also independent of the initial film thickness. The initial film thickness was found experimentally to have no effect on the final thickness by several investigators (Lai, 1979; Daughton and Givens, 1982).
When evaporation is negligible, the general twoterm expansion of solution, eq. (14), indicates that the film contour is dependent on the initial function of film profile, provided by the delivery system or developed in the spin-up process, multiplying by a time factor of $(3 /(3+4 T))^{3 / 2}$. This time factor and the initial profile function

$$
g\left(\left(\frac{3}{3+4 T}\right)^{3 / 4} R\right)
$$

decrease with increasing time and finally approaches zero at long spinning times. Therefore the film becomes more uniform when time is increased and thus finally smooths out when time is long enough to make the second term vanish. The comparisons of the exact numerical and approximate perturbation solutions in three-term expansion for tested linear and sinusoidal film profiles are shown in Figs 2 and 3, respectively. It can be seen from the figures that the exact solution and approximate solution for each case always coincide together. So the approximate solutions by the perturbation method is accurate enough to represent the dynamic film profile in the spin-off process.

In the case of evaporation and uniform initial film distribution, the exact solution is formulated by eq. (19), which is complicated and virtually implicit on film thickness and thus inconvenient to use. The perturbation solution in three-term expansion, eq. (25), is now formulated by using the evaporation parameter $\varepsilon^{\mathbf{3}}$ as a perturbation quantity. Figure 4 shows film thickness calculated from the exact numerical solution and the three-term approximate solution vs the perturbation quantity by employing dimensionless time as a parameter. As can be seen from Fig. 4, the film thickness calculated from the perturbation solution and exact solution is almost the same. So the perturbation solution can be used to predict the film thickness at any time in the case of small evaporation. At slightly high evaporation, this perturbation solution gradually has a small deviation from the exact solution and the difference slightly deteriorates with increasing evaporation parameter $\varepsilon^{3}$. For nonuniform initial profiles, we can use two perturbation quantities to characterize the effect of evaporation and the radial variation of initial thickness on the film thickness and uniformity. Here we still use a linear and a sinusoidal function to illustrate the deviation of the second-term approximate solution from the exact solution, as seen in Figs 5 and 6. From these figures, the approximate solutions to eq. (7) subject, respectively, to linear function, eq. (27), and sinusoidal function, eq. (28), are almost the same as the exact solutions which are obtained by the numerical method. As can be seen from Figs 2 and 3 or Figs 5 and 6, it can be found that the film thickness will be independent of the initial film contour as the spinning time is long enough and finally smooths out with respect to the initial nonuniformity of the film if the evaporation is small. The approximate three-term expansion indicates that only the first- and second-order terms are related to 


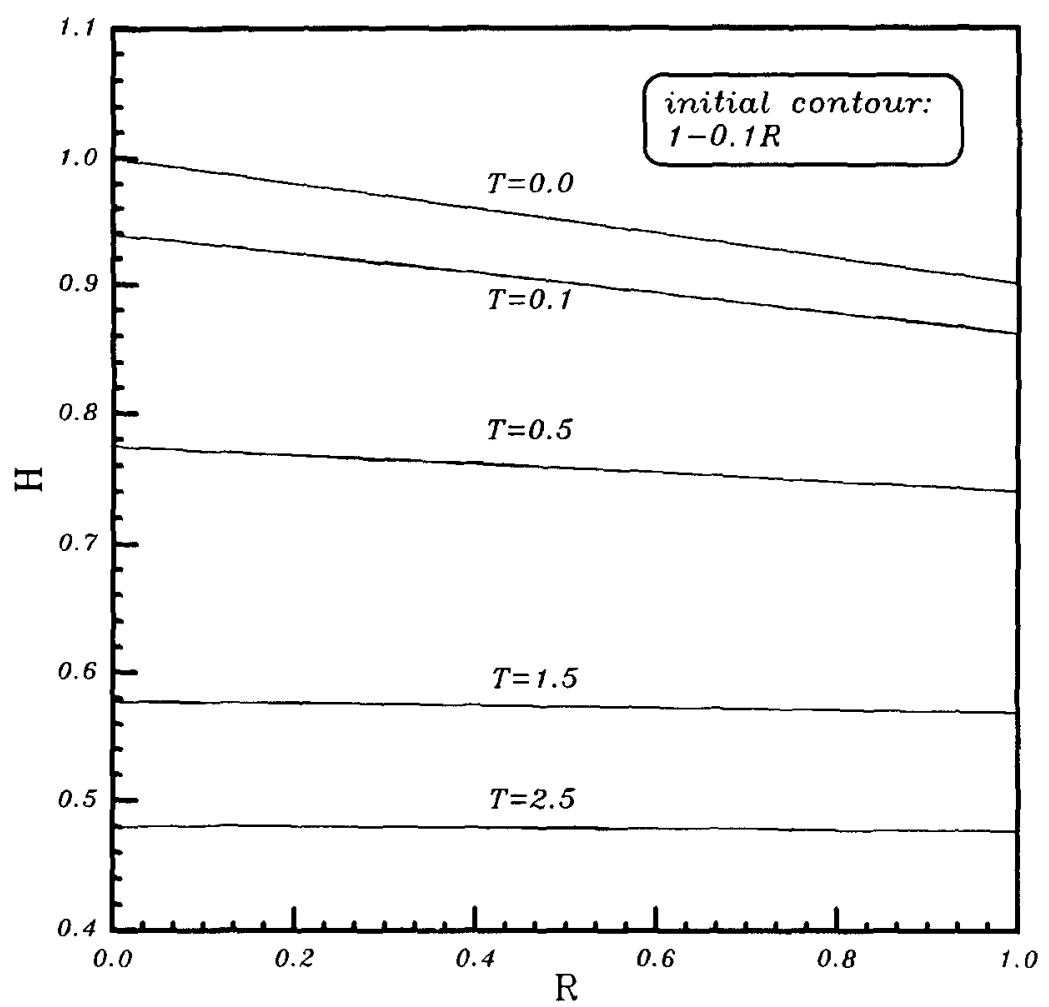

Fig. 2. Dimensionless film thickness profile; initial surface contour; $1-0.1 R$; ( $\longrightarrow$ ) exact solution; $(--)$ approximate solution.

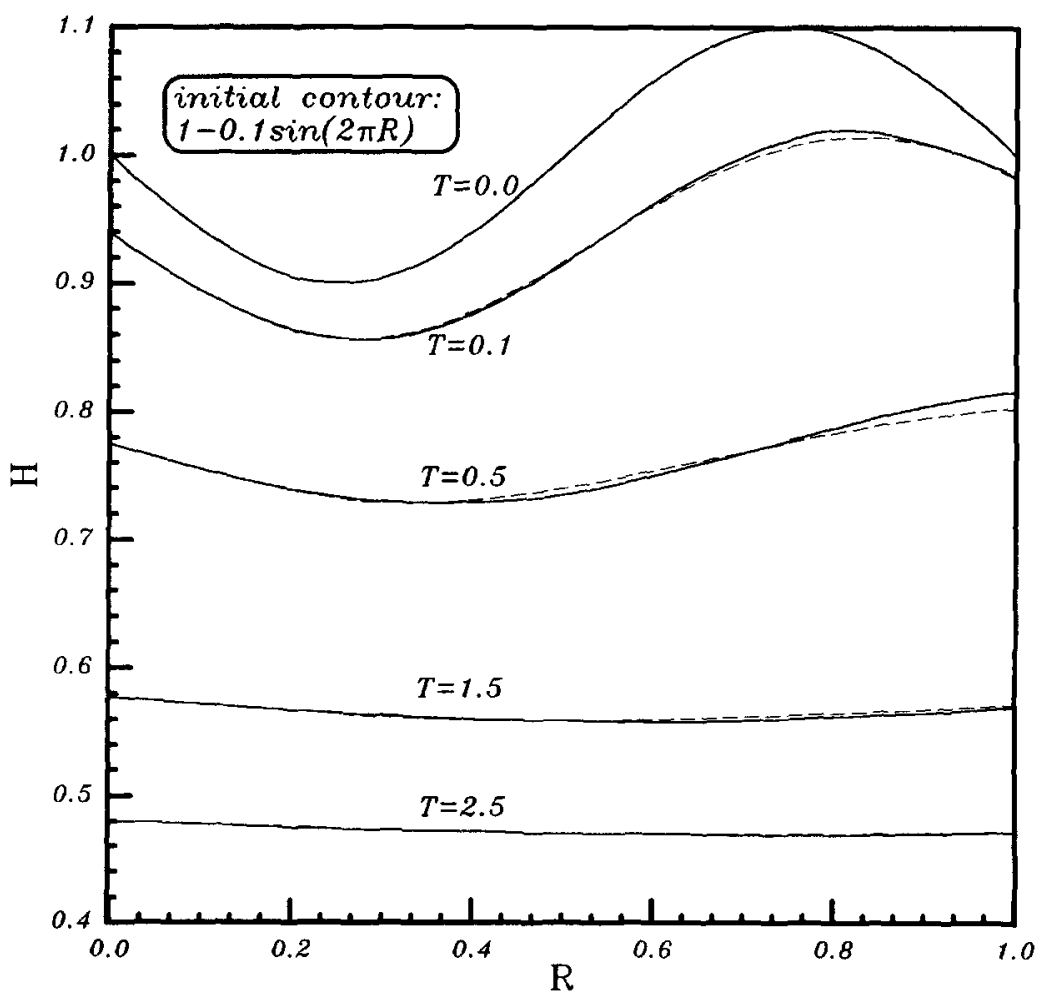

Fig. 3. Dimensionless film thickness profile; initial surface contour: $1-0.1 \sin (2 \pi R)$; $(-$ exact solution; (- - ) approximate solution. 


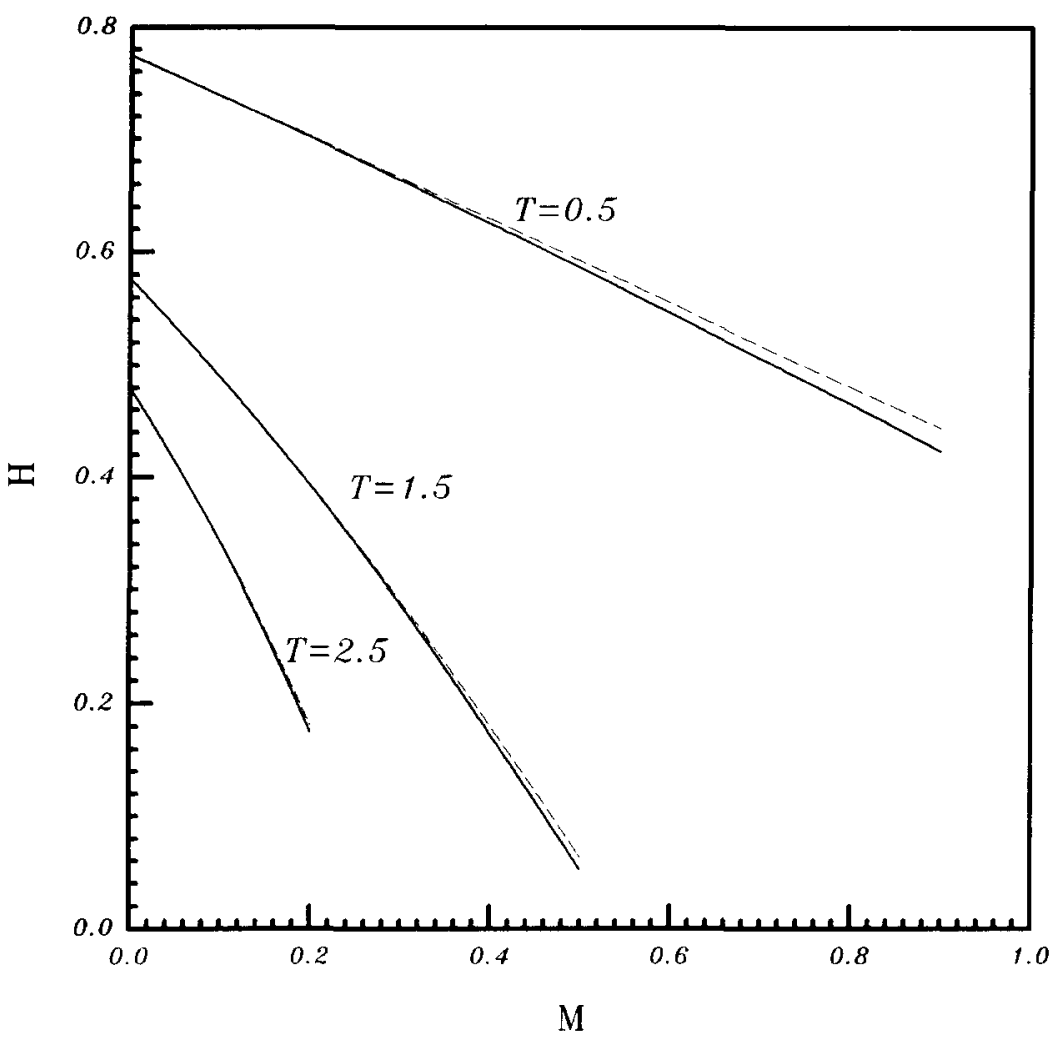

Fig. 4. Dimensionless film thickness vs $M\left(=\varepsilon^{3}\right)$ at various times with uniform initial thickness.

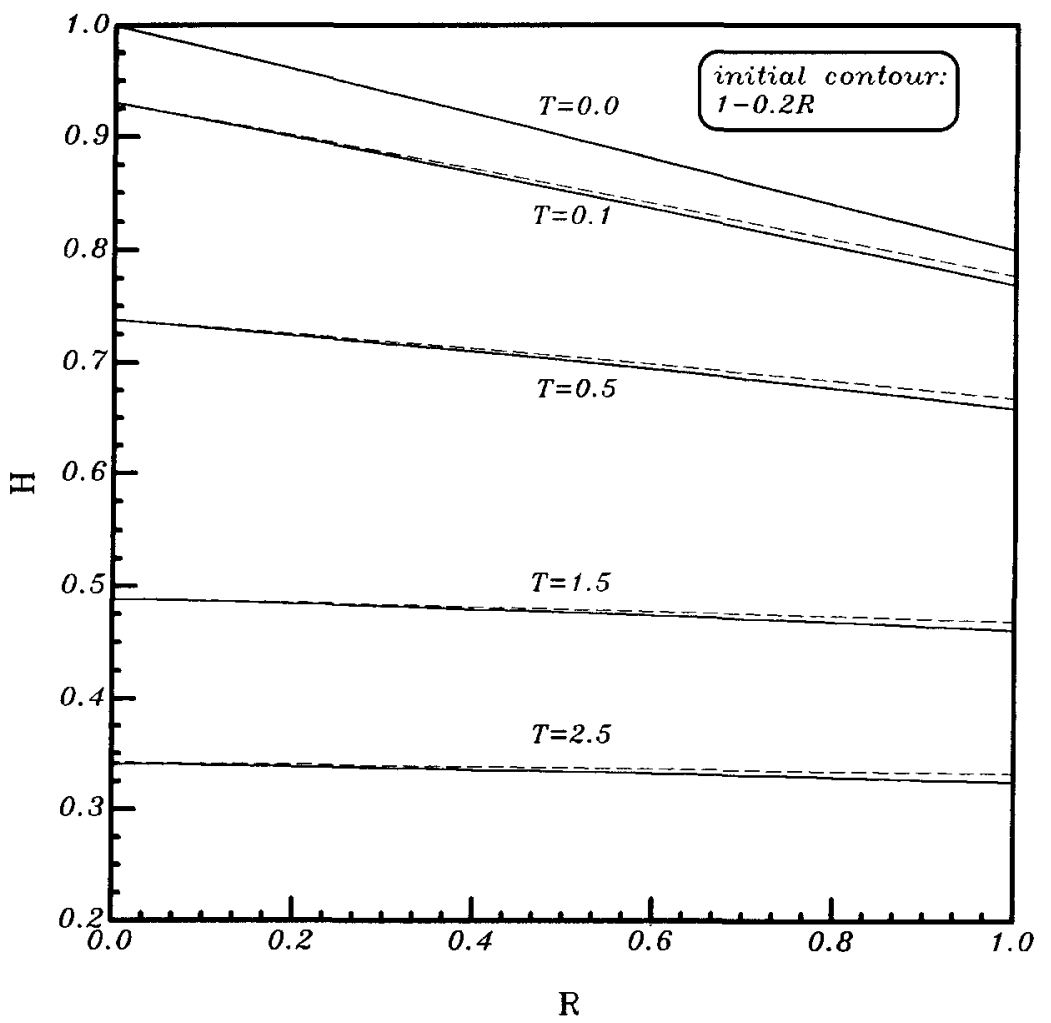

Fig. 5. Dimensionless film thickness profile with evaporation; initial surface contour: $1-0.2 R$; the evaporation parameter $M=0.1 ;(-)$ exact solution; $(--)$ approximate solution. 


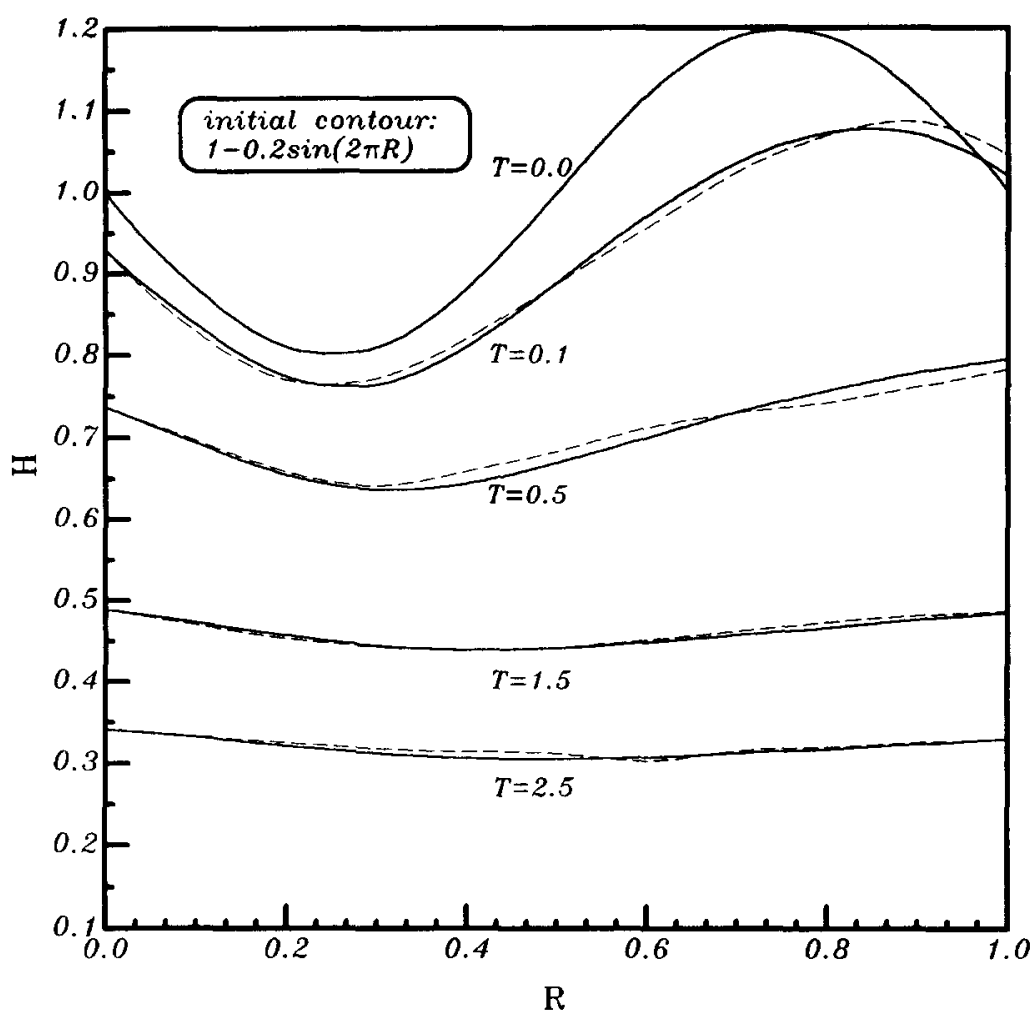

Fig. 6. Dimensionless film thickness profile with evaporation; initial surface contour; $1-0.2 \sin (2 \pi R)$; the evaporation parameter $M=0.1$; ( $)$ exact solution; (- - ) approximate solution.

the radial distribution, so the film will be more uniform as the evaporation parameter $\varepsilon^{3}$ gets smaller. The degree of film uniformity with respect to the average dynamic film thickness can be defined as

$$
\mathrm{DU} \%=100 \%-\frac{H_{\max }-H_{\min }}{1 / 2\left(H_{\max }+H_{\min }\right)} \times 100 \%
$$

where $H_{\max }$ and $H_{\min }$ represent the dynamic maximum and minimum values of film thickness, respectively. The effect of evaporation in spin coating on the film uniformity can be investigated by plotting the degree of uniformity vs time for various evaporation parameters $M\left(=\varepsilon^{3}\right)$. This is shown in Figs 7 and 8 for a linear and a sinusoidal initial film profile, respectively. As can be seen from the figures, the degree of uniformity is better for spin coating without evaporation and gets worse with larger uniform evaporation with nonuniform initial profile. The thinning rate of the spin-coating film increases with increasing evaporation. Thus it needs less time to reach a fixed film thickness with evaporation, but the film uniformity is sacrificed. For the case of small evaporation, as already shown in the theoretical analysis, the radial film-thickness variation will finally vanish at sufficient spinning time, and a uniform film on the disc is obtained. It must be noted that the degree of uniformity is defined to be related with the dynamic film thickness. Thus if a difference between maximum thickness and minimum thickness [i.e. the numerator of the second term in eq. (31)] is the same, the smaller average thickness [i.e. the denominator of the second term in eq. (31)] make the degree of uniformity worse. This is for the cases of large evaporation. The average film thickness decreases rapidly due to the effect of uniform evaporation on the film thinning and thus the denominator of the degree of film uniformity becomes smaller with the time evolution. However, the uniform evaporation does not have a significant effect on the change of radial film thickness and thus the numerator is nearly the same as the case of small evaporation. Therefore the quotient in the second term will have a minimum and the dynamic degree of uniformity goes through a maximum at some given time. A critical evaporation parameter $M_{C}$ can be estimated theoretically from eq. (22), which is $M_{C}=0.341$ with $H_{\text {min, initial }}=0.8$ and $M_{C}=0.486$ with $H_{\text {min, initial }}=0.9$. Figures 7 and 8 represent, respectively, the results of the dynamic degree of film uniformity from the numerical computation of the linear and sinusoidal film profiles at various evaporation parameter $M$. As can be seen from Figs 7 and 8 , the film uniformity can be improved in the spinning process if $M$ is less than the critical value. Thus, for obtaining a more uniform film on a spinning disc, we can get a criterion from eq. (8), which indicates the evaporation effect should be suppressed by formulating the solution recipe with low vapor pressure solvents, or increasing the spinning speed, and employing a thicker initial film. 


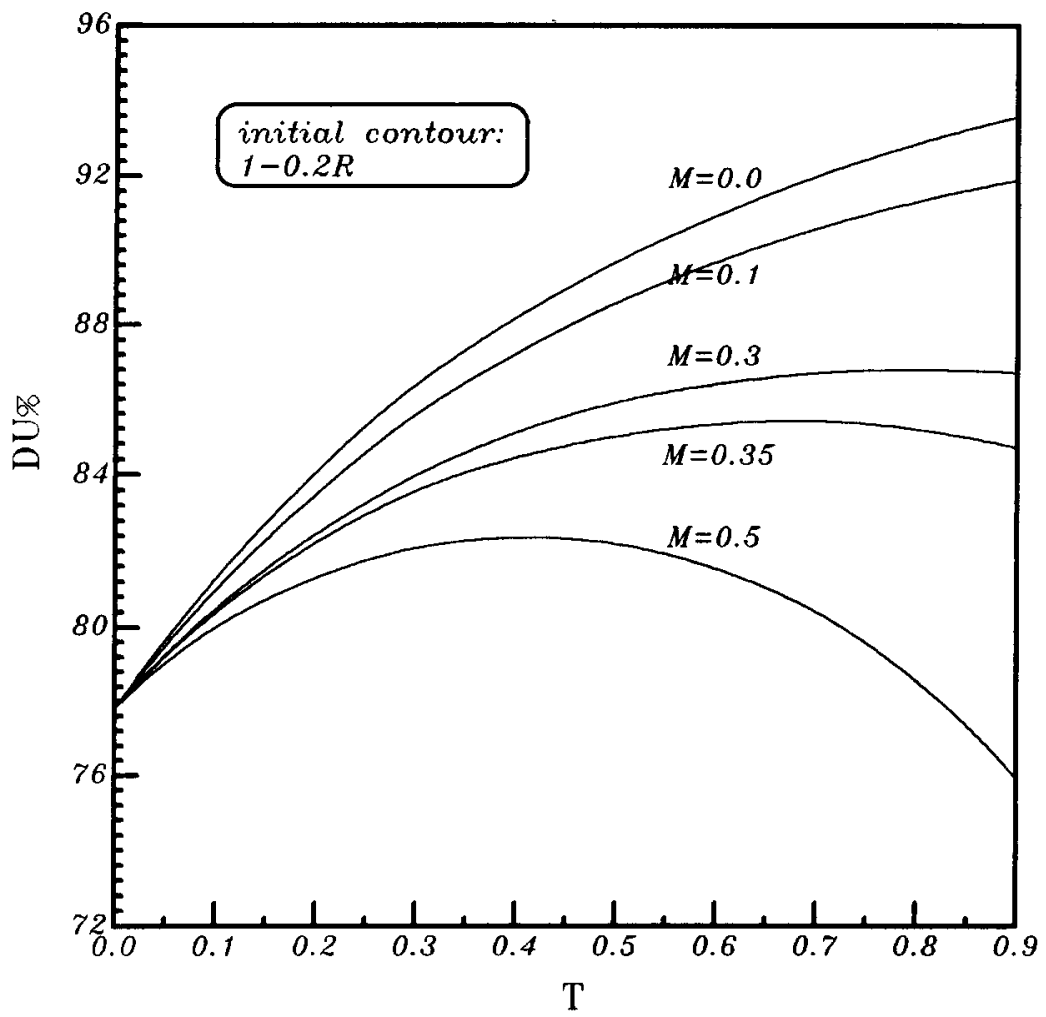

Fig. 7. Degree of film uniformity vs dimensionless time at different values of the evaporation parameter; initial film contour is $H=1-0.2 R$.

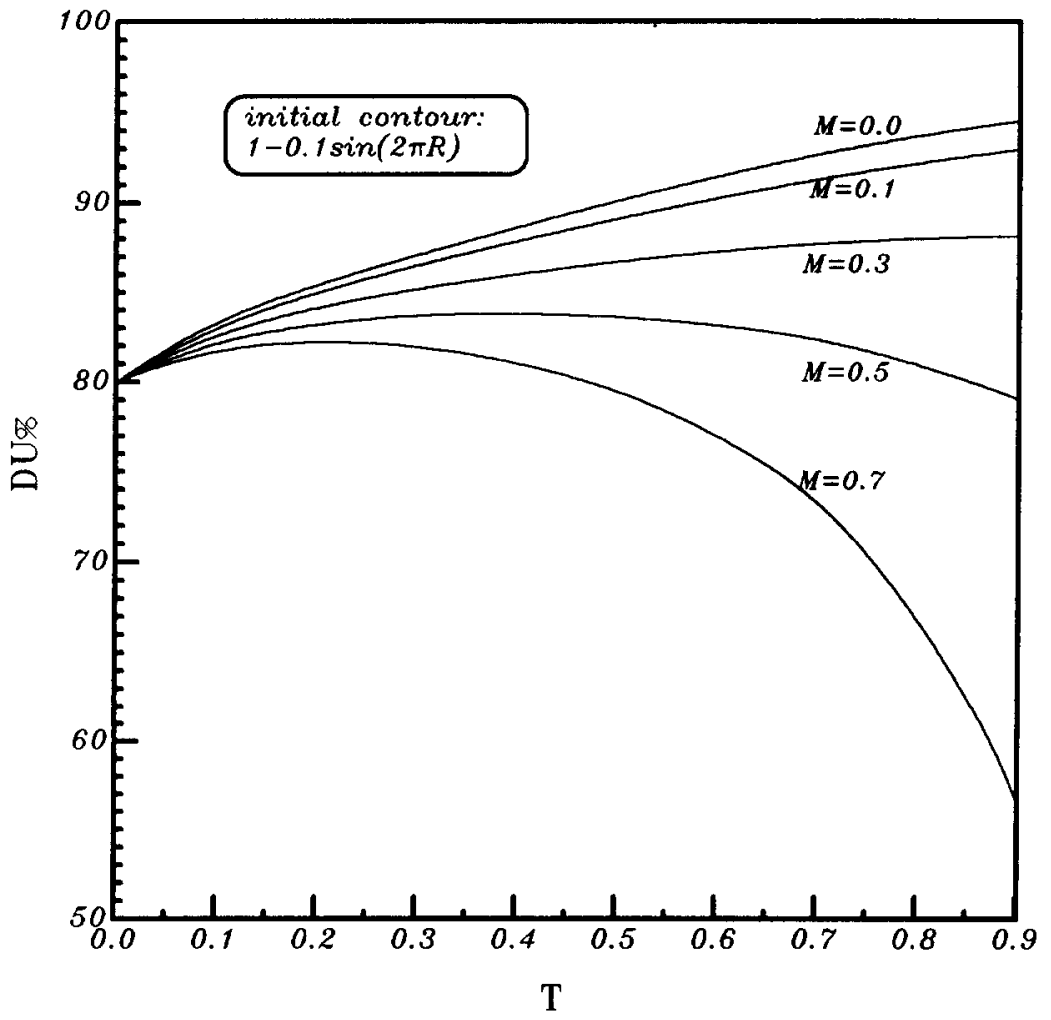

Fig. 8. Degree of film uniformity vs dimensionless time at different values of the evaporation parameters; initial film contour is $H=1-0.1 \sin (2 \pi R)$. 


\section{CONCLUSION}

The dynamic film uniformity of a Newtonian fluid in spin coating with or without evaporation in axisymmetric free surface flow on a spinning disc was elucidated analytically. Perturbation technique is used to solve the governing equation and to obtain the analytical explicit solution for each case. The approximate solution in three-term expansion by perturbation method is proved to be accurate enough to represent the film profile. It was found that the evaporating or nonevaporating film is always uniform across the flat spinning disk if the initial film distributes uniformly in thickness. A nonuniform initial film can be finally smoothed out after a sufficient spinning time, if its evaporation rate is smaller in comparison with the criterion we proposed in the model. Thus, for obtaining a more uniform film on a spinning disc, evaporation effect should be suppressed by formulating the solution recipe with low vapor pressure solvents, or increasing the spinning speed, and employing a thicker initial film.

\section{NOTATION}

$h$ film thickness, $\mathrm{m}$

$h_{0} \quad$ reference initial film thickness, $\mathrm{m}$

$H$ dimensionless film thickness

$m$ mass flux due to evaporation, $\mathrm{kg} / \mathrm{m}^{2} / \mathrm{s}$

$M$ dimensionless mass flux due to evaporation

$q$ volumetric flow rate per unit circumference, $\mathrm{m}^{2} / \mathrm{s}$

$r_{0} \quad$ radius of the spinning disc, $m$

$R$ dimensionless radial coordinate

$t$ time, $\mathbf{s}$

$T$ dimensionless time

$v_{r}$ radial velocity, $\mathrm{m} / \mathrm{s}$

$v_{\theta}$ azimuthal velocity, $\mathrm{m} / \mathrm{s}$

\section{Greek letters}

$\varepsilon \quad$ one third power of dimensionless mass flux

$\mu$ solution viscosity, $\mathrm{kg} / \mathrm{m} / \mathrm{s}$

$\xi \quad$ a small quantity used in perturbation technique

$\rho$ solution density, $\mathrm{kg} / \mathrm{m}^{3}$

$\tau \quad$ shear stress, $\mathrm{N} / \mathrm{m}^{2}$

$\omega$ angular velocity, $\mathrm{rad} / \mathrm{s}$

\section{REFERENCES}

Acrivos, A., Shah, M. and Petersen, E. E., 1960, On the flow of non-Newtonian liquid on a rotating disk. J. Appl. Phys. 31, 963-968.
Adamson, A. W., 1982, Physical Chemistry of Surfaces, 4th Edition. Wiley, New York.

Aris, R. and Amundson, N. R., 1973, Mathematical Methods in Chemical Engineering, Vol. 2. Prentice-Hall, Englewood Cliffs, NJ,

Bornside, D. E., Maccsko, C. W. and Scriven, L. E., 1987, On the modeling of spin coating. J. Imaging Technol. 13, 122-130.

Bornside, D. E., Maccsko, C. W. and Scriven, L. E., 1989 , Spin coating-one dimensional model. J. Appl. Phys. 66, $5185-5193$.

Daughton, W. J. and Givens, F. L., 1982, An investigation of the thickness variation of spun-on thin-films commonly with the semiconductor industry. J. Electrochem. Soc. 129, 173-179, 2811-2813.

Elliott, D. J. and Hockey, M. A., 1979, One micron range photoresist imaging: a practical approach. Solid State Technol. 22, 53.

Emslie, A. G., Booner, F. T. and Peck, L. G., 1958, Flow of a viscous liquid on a rotating disk. J. Appl. Phys. 29, 858-862.

Flack, W. W., Soong, D. S., Bell, A. T. and Hess, D. W., 1984, A mathematical model for spin coating of polymer resists. J. Appl. Phys. 56, 1199-1206.

Higgins, B. G., 1986, Film flow on a rotating disk. Phys. Fluids 29, 3522-3529.

Jenekhe, S. A. and Schuldt, S. B., 1984, Coating flow of non-Newtonian fluids on a flat rotating disk. Ind. Engng Chem. Fundam. 23, 432-436.

Jenekhe, S. A. and Schuldt, S. B., 1985, Flow and film thickness of Bingham plastic liquids on a rotating disk. Chem. Engng Commun. 33, 135-147.

Lai, J. H., 1979, A investigation of spin coating of electron resists. Polym. Engng Sci. 19, 1117-1121.

Lawrence, C. J., 1988, The mechanics of spin coating of polymer films. Phys. Fluids 31, 2786-2795.

Lawrence, C. J., 1990, Spin coating with slow evaporation. Phys. Fluids A 2, 453-456.

Levich, V. G., 1962, Physiochemical Hydrodynamics, Prentice-Hall, Englewood Cliffs, NJ.

Meyerhofer, D. J., 1978, Characteristics of resist films produced by spinning. J. Appl. Phys. 49, 3393-3397.

Rehg, T. J. and Higgins, B. G., 1988, The effect of inertia and interfacial shear on a rotating disk. Phys. Fluids 31, 1360-1371.

Sukanek, P. C., 1985, Spin coating, J. Imaging Technol. 11, 184-190.

Sze, S. M., Ed., 1988, VLSI Technology, 2nd Edition, McGraw Hill, New York.

Thompson, L. F. and Bowden, M. J., Eds, 1983, Introduction to Microlithography, American Chemical Society, Washington, D.C.

Yen, S. C. and Wang, J. S., 1992, Mass transfer and fluid flow due to a rotating disk with external forced convection. Chem. Engng Sci. 47, 2001-2008.

Washo, B. D., 1977, Rheology and modelling of the spin coating process. IBM J. Res. Dev., 190-198, 21. 